\title{
Role of Intraperitoneal Drainage after Laparoscopic Sleeve Gastrectomy
}

\author{
Mohamed A. Marzouk, MD; ${ }^{1}$ Samy Gamil Akhnoukh, MD; ${ }^{1}$ Mohamed Rady, MD; ${ }^{1}$ Haytham \\ Mohamed Nasser, MD² \\ ${ }^{1}$ General Surgery Department, Ain Shams University, Cairo, Egypt \\ ${ }^{2}$ Radiology Department, Ain Shams University, Cairo, Egypt
}

Background and aims: Laparoscopic sleeve gastrectomy (LSG) is a very popular bariatric procedure. Intraperitoneal bleeding, haematoma and staple line leak are the most common postoperative complications of LSG. Many surgeons routinely leave an intraperitoneal drain (IPD) thinking it will help in managing complications. Our aim was to evaluate the role of IPD in diagnosing and treating post-operative complications after sleeve gastrectomy.

Patients and methods: We collected the data of patients who underwent primary LSG at our department between February 2016 and March 2018. 120 patients (group A) had LSG with positioning of IPD and 105 patients (group B) had LSG without IPD. All operations were performed by the same operative team and were completed laparoscopically. Inclusion criteria were a body mass index (BMI) greater than $40 \mathrm{~kg} / \mathrm{m} 2$ or greater than $35 \mathrm{~kg} /$ $\mathrm{m} 2$ accompanied with relevant co-morbidities. Exclusion criteria were the inability to undergo general anesthesia, patients with previous bariatric procedures, patients with previous intragastric balloon, patients on anticoagulation and patients older than 60 years.

Results: The two groups were not different in their outcome. In Group A, 3(2.5\%) patients had bleeding ( only one was evident in the drain) managed conservatively, one $(0.8 \%)$ of them later on had surgical bed haematoma treated by $\mathrm{U} / \mathrm{S}$ guided aspiration and pigtail insertion. Also in group $\mathrm{A}$, one case $(0.8 \%)$ of leakage managed by endoscopic stent and laparoscopic lavage and draining. In Group B, 4 cases (3.8\%) had bleeding managed conservatively, 2 of them $(1.9 \%)$ had haematoma later on managed by $\mathrm{U} / \mathrm{S}$ guided aspiration and pigtail insertion. No leakage occurred in group $B$.

Conclusions: Insertion of drains does not help in diagnosing or treating post-LSG complications as bleeding, leakage or haematoma.

Key words: Laparoscopic Sleeve, Intraperitoneal drain (IPD).

\section{Introduction}

Laparoscopic sleeve gastrectomy (LSG) was known in 1990 as part of biliopancreatic diversion with duodenal switch. ${ }^{1}$ In the early 2000s, it became a primary procedure for treatment of morbid obesity. ${ }^{2}$

Its main advantages include: No interruption of gastro-intestinal continuity, no foreign body, no malabsorption, and possibility of conversion to multiple bariatric procedures. Mid-term results are generally good, with some reports about weight regain after 3 years. ${ }^{3}$

Postoperative complications included intraperitoneal bleeding, surgical bed haematoma, abscess formation, and staple line leak. ${ }^{4}$ The leak rate can vary between $1 \%$ and $3 \%$ for LSG, and the risk of postoperative bleeding has been reported to be between $1 \%$ and $6 \%$ in different series. ${ }^{5}$ One remarkable question is if intraperitoneal drains (IPD) may facilitate the identification and treatment of these complications. This retrospective study was done to assess the utility of routine placement of intraperitoneal drains LSG in detection and management of these postoperative complications.

\section{Patients and methods}

We collected the data of 225 cases, who underwent primary LSG at our Department. The idea of no IPD was introduced in November 2017.6 All patients treated between February 2016 and November 2017 routinely had IPD inserted after LSG. Conversely, all patients operated from November 2017 until March 2018 did not have IPD. 120 patients (group A) had LSG with positioning of IPD and other 105 patients (group B) had LSG without IPD. All operations were performed by the same operative team and were completed laparoscopically. Inclusion criteria were a body mass index (BMI) greater than $40 \mathrm{~kg} / \mathrm{m} 2$ or greater than $35 \mathrm{~kg} / \mathrm{m} 2$ accompanied with relevant co-morbidities. Exclusion criteria were the inability to undergo general anesthesia, patients with previous bariatric procedures, patients with previous intragastric balloon, patients on anticoagulation and patients older than 60 years.

Routine preoperative investigations included complete blood picture, bleeding profile, thyroid 
profile, liver and kidney functions, viral markers, pelvi-abdominal ultrasound, chest $X$-ray and echocardiography. Anti-embolism stockings were provided and patients were required to urinate before transportation to operative room to abandon need for urine catheters.

All patients received a dose of low molecular weight heparin (LMWH) subcutaneously 12 hours before surgery and 12 hours after surgery continued to 2 weeks postoperatively. Complete fasting was advised 8 hours prior to surgery. All patients were admitted to the hospital the morning of surgery. In Group A, IPDs were removed at second postoperative day after oral fluids.

\section{Operative technique}

In our department we used five trocars technique; three $12 \mathrm{~mm}$ and two $5 \mathrm{~mm}$, and a $10-\mathrm{mm} 30^{\circ}$ scope. For an energy source, we used LigaSure ${ }^{\mathrm{TM}}$ (Medtronic). The patient; after general anesthesia, was placed in anti-Trendeleburg position. Pneumoperitoneum was induced using a Veress needle. First trocar into peritoneal cavity was an optical trocar. The LSG started with the interruption of the great gastric curvature vessels, dissecting the gastrocolic and gastrosplenic ligaments near the stomach. A 39-Fr boogie was inserted in the stomach and using a stapler a longitudinal gastrectomy was performed starting $2-4 \mathrm{~cm}$ proximal to the pylorus and excising completely the gastric fundus. In all procedures, we used long Endo GIA Universal Stapler $^{\mathrm{TM}}$ (Medtronic) $60 \mathrm{~mm}$ starting with a green cartridge followed by blue ones. We routinely reinforced staple line with clips, sutures and surgicel to reduce the rate of staple line bleeding. In group A, a non-suction drain (Nelaton catheter) was left along the staple line. In group B no drain was left. Methylene blue test was done for leakage at the end of operation. Blood pressure was elevated to at least $140 \mathrm{~mm} \mathrm{Hg}$ at the end of the operation. Patients resumed clear fluids on $1^{\text {st }}$ postoperative day (POD) and were discharged on the 1st or 2nd POD, unless there was a complication. We followed patients for at least 3 months to assess postoperative development of early postoperative complications. Leakage was suspected if there is persistent fever, tachycardia and abdominal pain. If suspected CT scan with contrast was done. Bleeding was recorded as presence of melena or hematemesis or blood in the drain, tachycardia, hypotension and drop of hemoglobin more than $2 \mathrm{gm} / \mathrm{dl}$. If postoperative haematoma was suspected, broad spectrum strong parenteral antibiotic is started and pelvi-abdominal $\mathrm{U} / \mathrm{S}$ was done with aspiration of haematoma and pigtail insertion if needed.

U/S was done using GE logiq p5 ultrasound machine with aspiration of haematoma using angiocath 14 gauge and/or pigtail insertion if needed using (Dawson-Mueller Multipurpose Drainage Catheter, Cook Medical.)

\section{Results}

There were no significant difference between the 2 groups as regards the demographic features (Table 1).

Table 1: Demographic Features

\begin{tabular}{lcc}
\hline & Group A & Group B \\
\hline Age (mean, range) & $38(20-58)$ & $36(18-57)$ \\
Male: Female & $29: 91$ & $18: 87$ \\
BMI (mean ,range) & $46(39-52)$ & $43(37-49)$ \\
Comorbidities & & 19 \\
- Hypertension $\quad$ 15 & 22 & 27 \\
- DM & 5 & 7 \\
- Dyslipidaemia & $52(30-65)$ & $35(25-45)$ \\
\hline
\end{tabular}

As regards postoperative complications (Table 2); 3 cases of intraperitoneal bleeding occurred in Group A and were conservatively treated. One of them presented with evident active bleeding in the drain about 500 cc with drop of haemoglobin by $2 \mathrm{gm} / \mathrm{dl}$ and was managed conservatively by packed RBCs transfusion and the patient was discharged on 4th POD on broad spectrum IV antibiotic. The other 2 cases presented only with tachycardia and drop of the haemoglobin level but there was no evident bleeding in the IPD. Both these 2 cases were managed by packed RBCs transfusion; one unit in one case, and two units in the other case. In group B; 4 patients developed postoperative bleeding diagnosed by tachycardia and drop of haemoglobin level. They were managed conservatively and only two patients required transfusion of 2 units of packed RBCs.

Routine pelvi-abdominal U/S was done by an 
experienced radiologist for any patient who developed postoperative persistent fever, abdominal pain, or left shoulder pain.

In group $A$, 4 patients needed $U / S ; 2$ of them had persistent abdominal pain and 2 had persistent fever. Two patients was found to have chest infection and was managed by broad spectrum antibiotics. One patient was found to have surgical bed haematoma where aspiration and pigtail insertion was done (Figure 1). Pigtail was removed after 5 days. One other patient was found to have purulent collection where pig tail was inserted (Figure 2).

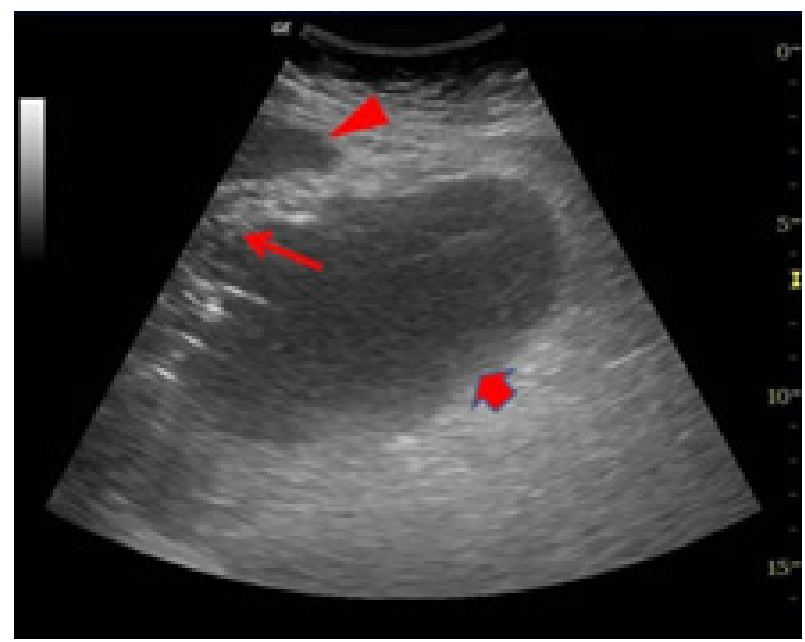

Fig 1: U/S showing haematoma Wide arrow: left lobe of the liver Narrow arrow: head gastric pouch.

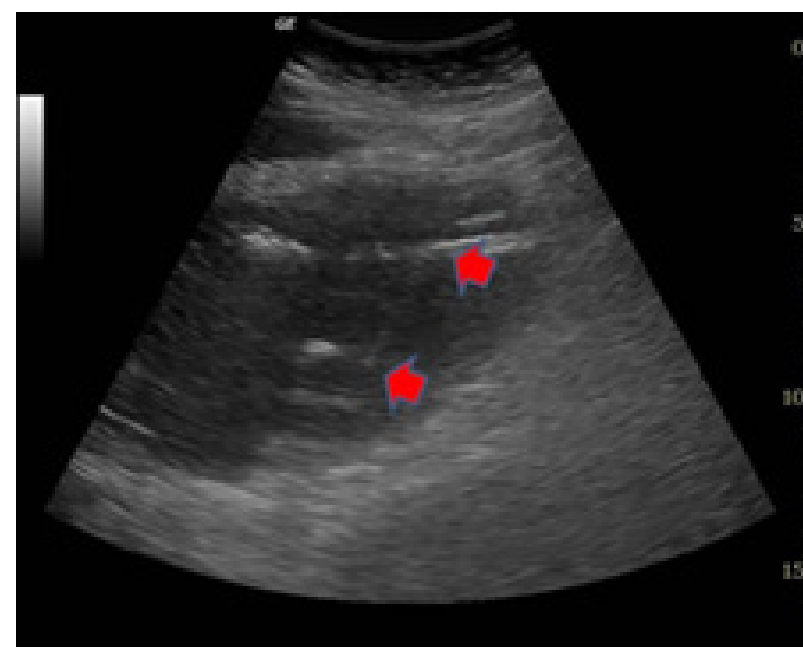

Fig 2: U/S showing pigtail inside the collection.

Pelvi-abdominal CT scan with oral contrast was done and there was a small leak at the stomach body (Figure 3). Simultaneous laparoscopic peritoneal lavage with drain insertion and endoscopic Mega stent insertion was done (Figure 4). The patient was discharged after 3 days and the stent was removed after 4 weeks.

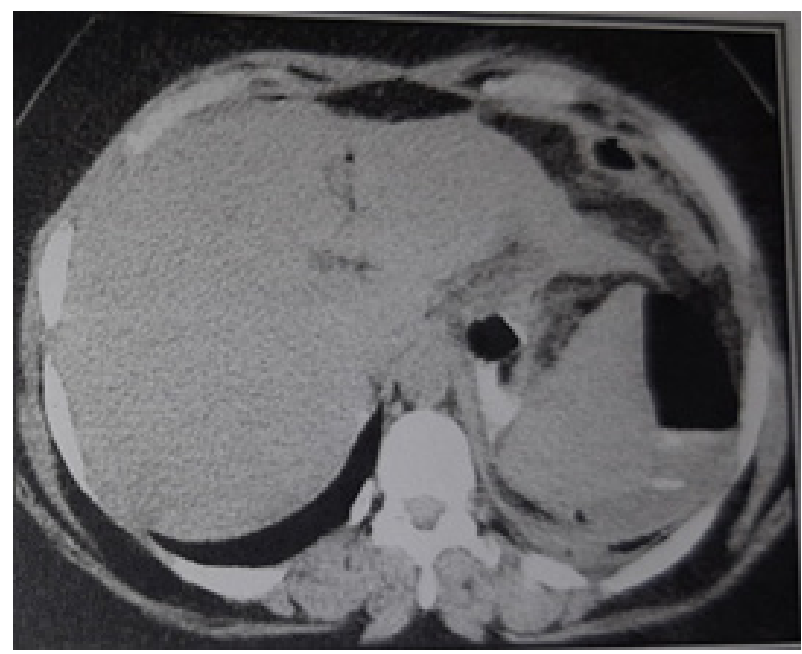

Fig 3: CT showing leakage.

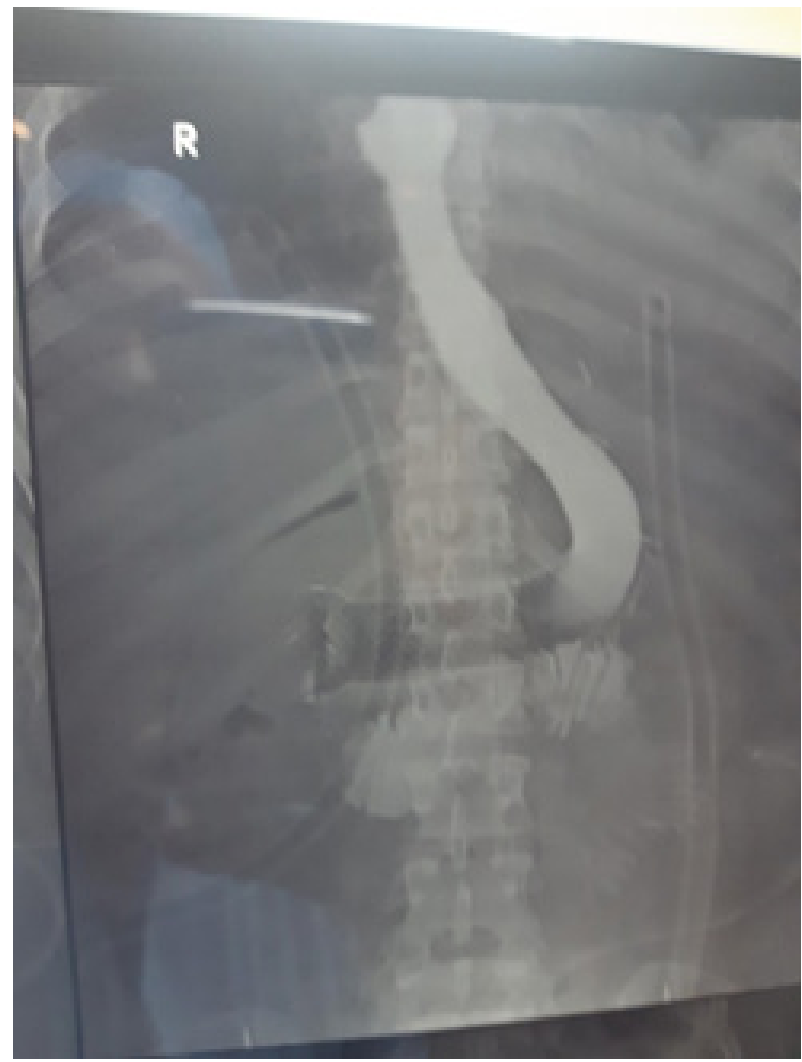

Fig 4: Mega stent placed.

In group B 3 patients were presented with postoperative fever and left shoulder pain. They all did an U/S where 2 of them had surgical bed haematoma and aspiration with pigtail insertion were done. The third patient was discovered to have chest infection. No cases of leakage were discovered in group B. No patients needed re-operation. 
Table 2: Postoperative complications

\begin{tabular}{lcc}
\hline & $\begin{array}{c}\text { Group A } \\
\text { (n 120) }\end{array}$ & $\begin{array}{c}\text { Group B } \\
\text { (n 105) }\end{array}$ \\
\hline Complications & 3 & 4 \\
Bleeding & 1 & 2 \\
Haematoma & 1 & 0 \\
Leakage & 2 & 1 \\
Chest inf. & 0 & 0 \\
Reoperation & & \\
\hline
\end{tabular}

\section{Discussion}

Complications in morbidly obese patients are usually difficult to diagnose. Clinical signs are not usually obvious and may be the only sign of a possible complication is low fever or tachycardia. Some surgeons; from their point of view, persist on using drains to provide more safety in the postoperative care of morbidly obese patients, even though the utility of drain placement in general surgery and in bariatric patients in particular remains controversial. ${ }^{6,7}$

Nowadays, many surgeons perform a large number of LSG as it is a simple and effective procedure. But its complications are not easy to manage. Staple line leaks, and surgical bed haematoma are the most commonly complications, ranging in different series between $1 \%$ and $3 \%{ }^{8}$

Postoperatively, drains can sometimes be helpful in detecting bleeding. This does not occur in all cases of intraperitoneal bleeding. Heart rate and drop of haemoglobin level are very important in diagnosing bleeding. With these tools, all patients were managed safely, even in these without drains, so there was no role for drains in detecting or managing bleeding. When bleeding was identified, conservative management of stopping anticoagulation with fluid and blood transfusion was sufficient in most of cases. Drain placement did not seem to protect from the formation of a haematoma since results were nearly similar between the two groups. ${ }^{9}$

Leaks occur in $1-3 \%$ of LSG cases. It is the major complication of LSG, resulting in peritonitis, multisystem organ failure, and may lead to mortality. ${ }^{10}$ Early detection of leaks is proven to reduce morbidity and mortality. However, diagnosing leaks is not easy. Obesity makes clinical limitations, as diagnosis of peritonitis in these patients is very difficult. Patients who are super obese cannot get into a CT scan bed.?

In our study only one patient developed leak. The patient had tachycardia, fever, leukocytosis and high CRP levels. Methylene blue test was negative, suggesting that diagnosis of leak cannot be based on the evaluation of the drain liquid. Also the leak may be late after removal of the drain. CT scan and UGI with contrast study was important to confirm the diagnosis of leakage.

In our study we were aiming to find the role of IPD in diagnosis of surgical complication of LSG and their conservative management.

Many surgeons prefer using IPD after LSG as drains can diagnose intraperitoneal bleeding, early leaks and prevent formation of haematoma. Bleeding may be intra- or extra luminal. Intraluminal bleeding from the staple line usually presents with an upper gastrointestinal bleed. It is presented by hematemesis or melena. Intraperitoneal bleeding is usually from the staple line, dissected greater omentum, short gastric vessels, liver injury or trocar sites. ${ }^{11}$ Many sealing materials are commercially available to decrease the staple line bleeding but their use remains controversial. Albanopoulos et al. ${ }^{7}$ did not observe a significant difference in their rate of postoperative bleeding in patients with staple line suturing. Curro et al. ${ }^{11}$ also do not routinely use any reinforcement materials (sutures or buttresses) for LSG.

Moreover, as already described by Albanopoulos K. et al. ${ }^{7}$ and Curro et al. ${ }^{11}$ and confirmed in our study, most cases of intraperitoneal bleeding or leakage, drainage are silent and did not help in diagnosing complications which are often revealed by other clinical signs laboratory investigations or by diagnostic imaging. Only one case in our study IPD diagnosed bleeding.

In gastric surgery, the aim of drains was removal of fluid collections and early detection of postoperative complications. In our study drains had no role in diagnosing or managing complications.

The rapid progress in interventional radiology techniques as $\mathrm{U} / \mathrm{S}$ or $\mathrm{CT}$ guided percutaneous drainage and aspiration allowed surgeons to deal with post-LSG complications without surgical intervention and with low morbidity. ${ }^{(12,13)}$ Endoscopic intervention by stents, clips or plugs also is a very important tool in managing complications and decreasing morbidity and mortality.

\section{Conclusion}

Insertion of drains does not usually help in diagnosing or treating post-LSG complications as bleeding, leakage or haematoma. Also they don't exclude the intervention for these complications. Surgeons do not know during the operation which patient could benefit from the placement of a drain. 
Maybe patients undergoing revisional surgeries could benefit from a drain left for more than 3 days.

\section{Conflict of Interest}

All Authors have no conflict of interest with any institution or product that is mentioned.

\section{References}

1. Marceau $\mathrm{P}$, Biron $\mathrm{S}$, Bourque RA, et al: Biliopancreatic diversion with a new type of gastrectomy. Obes Surg. 1993; 3(1): 29-35.

2. Gumbs A, Gagner M, Dakin G, et al: Sleeve gastrectomy for morbid obesity. Obes Surg. 2007; 17(7): 962-9.

3. Himpens J, Dobbeleir J, Peeters G: Long-term results of laparoscopic sleeve gastrectomy for obesity. Ann Surg. 2010; 252(2): 319-324.

4. Chazelet $C$, Verhaeghe $P$, Perterli $R$, et al: Longitudinal sleeve gastrectomy as a standalone bariatric procedure: Results of a multicenter retrospective study. J Chir. 2009; 146: 368-372.

5. Rosenthal RJ, Diaz AA, Arvidsson $D$, et al: International sleeve gastrectomy expert panel consensus statement: Best practice guidelines based on experience of $>12000$ cases. Surg Obes Relat Dis. 2012; 8: 8-19

6. Dallal RM, Bailey L, Nahmias N: Back to basicsclinical diagnosis in bariatric surgery. Routine drains and upper GI series are unnecessary. Surg Endosc. 2007; 21: 2268-2271.
7. Albanopoulos $K$, Alevizos L, Linardoutsos D, et al: Routine abdominal drains after laparoscopic sleeve gastrcetomy: A retrospective review of 353 patients. Obes Surg. 2011; 21: 687-691.

8. Abou Rached A, Basile M, El Masri H: Gastric leaks post sleeve gastrectomy: Review of its prevention and management. World $\boldsymbol{J}$ Gastroenterol. 2014; 20(38): 13904-13910.

9. Fuks D, Verhaeghe $P$, Brehant $O$, et al: Results of laparoscopic sleeve gastrectomy: A prospective study in 135 patients with morbid obesity. Surgery. 2009; 145: 106-13.

10. Jacobs M, Bisland W, GomezE, et al: Laparoscopic sleeve gastrectomy: A retrospective review of 1 and 2 year results. Surg Endosc. 2010; 24: 781-785.

11. Curro G, Piscitelli G, Lazzara C, et al: Laparoscopic sleeve gastrectomy for morbid obesity: Role of intraluminal and intraperitoneal postoperative drainage. G Chir. 2017; 38(4): 181-184.

12. Téllez-Ávila F, Carmona-Aguilera GJ, ValdovinosAndraca $F$, et al: Postoperative abdominal collections drainage: Percutaneous versus guided by endoscopic ultrasound. Dig Endosc. 2015; 27(7): 762-766.

13. Chevaux JB, Deprez PH: Established EUSguided therapeutic interventions. Minerva Med. 2014; 105(5): 333-351. 